\title{
Estratégia Eficiente com Alta Disponibilidade para Provisionamento Dinâmico de Pontos de Acesso em Redes sem Fio de Grande Escala
}

\author{
Matheus B. de A. Rodrigues, Ana Carolina R. Mendes, Marcos Paulo C. de Mendonça, Gabriel R. Carrara, \\ Luiz Claudio S. Magalhães, Célio Vinicius N. de Albuquerque, Dianne S. V. Medeiros, Diogo M. F. Mattos \\ MídiaCom - IC/PPGEET/TCE/UFF \\ Universidade Federal Fluminense (UFF) - Niterói, RJ, Brasil
}

\begin{abstract}
Resumo-A associação dinâmica de usuários a pontos de acesso e o requisito de cobertura de ampla área tornam desafiador garantir a disponibilidade associada à eficiência energética em redes sem fio de larga escala. Este artigo propõe uma estratégia de provisionamento de pontos de acesso baseada em uma heurística de otimização de múltiplos objetivos. Os objetivos da heurística são maximizar a cobertura, garantindo alta disponibilidade, e minimizar o número de pontos de acesso ativos, melhorando a eficiência energética. A proposta é avaliada através da simulação de uma componente da rede sem fio da Universidade Federal Fluminense com 363 pontos de acesso. A simulação considera fluxos e traços reais de associação de usuários à rede. Os resultados mostram que a estratégia de melhor desempenho é a heurística gulosa que ativa pontos de acesso com o maior número de vizinhos potenciais, porém não ativados. A proposta reduz o número de usuários não servidos e o número de pontos de acesso ativos, assegurando a alta disponibilidade e eficiência energética com uma redução de até $84 \%$ no consumo total de energia.
\end{abstract}

Palavras-Chaves-Eficiência Energética, Disponibilidade, Rede Sem Fio, Campus Inteligente

\section{INTRODUÇÃO}

Em 2023 mais de 70\% da população mundial terá conexão móvel e a velocidade de conexão de dispositivos móveis triplicará [1]. Além disso, segundo o relatório anual da Cisco [1], o tráfego global de dados móveis para negócios crescerá seis vezes de 2017 a 2022, a uma taxa de crescimento anual de $42 \%$, com o número de pontos de acesso Wi-Fi crescendo quatro vezes de 2018 a 2023. Paralelamente, o gasto energético na transmissão de dados diminui em média pela metade a cada dois anos em países desenvolvidos [2]. A digitalização tem como contrapartida a exigência de maior uso da energia, anulando o efeito da otimização energética [3].

A redução do desperdício de energia é um fator importante para a utilização sustentável de recursos, mas é importante que essa redução não afete o desenvolvimento econômico [3], [4]. A inferência de padrões de uso de dados em redes sem fio permite definir medidas preventivas e de economia de energia. Nesse sentido, este artigo propõe e simula uma estratégia que visa otimizar o uso dos pontos de acesso (AP) em uma rede de grande escala, sem que haja perda de serviço e conectividade. A otimização aplicada considera o tempo ocioso da rede e explora os padrões de uso da rede sem fio da Universidade Federal Fluminense (UFF). Para a otimização de
APs ativos, um conjunto de dados é gerado a partir da coleta de dados de estatísticas de fluxos NetFlow, correlacionando estatísticas de fluxos de cada usuário ${ }^{1}$ da rede sem fio com características dos APs aos quais estão associados [5], [6]. Assim, é possível extrair agrupamentos de APs com áreas de cobertura semelhantes para finalmente identificar quais APs são essenciais em cada momento do dia em cada localização.

O objetivo deste artigo é solucionar o problema de ociosidade da rede Wi-Fi [7]. Na literatura, encontram-se exemplos de otimização da economia de energia. Lyu et al. [8] utilizam a previsão da carga e os dados dos padrões de uso da rede para desligar os APs pelo tempo previsto de ociosidade. O tempo de uso de cada cliente de celular nos APs é analisado utilizando uma máquina vetor de suporte (Support Vector Machine SVM). No entanto, esses métodos não são suficientes para fornecer a qualidade de serviço necessária aos usuários da rede. Ao considerar uma rede sem fio de grande escala e dinâmica, a otimização de apenas um objetivo prejudicaria a qualidade de serviço dos clientes [7]. O método de previsão de uso aplicado por Lyu et al. [8] é importante para identificar padrões e suas variações com o tempo. Com isso, a adição de um novo método para desligamento de APs inicialmente ligados é necessária para otimizar o funcionamento da rede, garantindo, assim, a eficiência energética e alto padrão de conectividade. Por sua vez, o presente artigo propõe uma estratégia de otimização de APs ativos com os objetivos de ampliar a cobertura e reduzir o número de APs ativos, um problema NP-difícil [9].

A Seção II discute os trabalhos relacionados. A Seção III apresenta a coleta de dados. A abordagem não supervisionada de agrupamento é exposta na Seção IV. A proposta é avaliada em uma rede sem fio de grande escala real e os resultados são discutidos na Seção V. A Seção VI conclui o trabalho.

\section{Trabalhos Relacionados}

O crescimento do conceito de Smart Campus vem beneficiando universidades no mundo todo, e não apenas na área tecnológica [10]. Uskov et al. [11] realizam a comparação de um Smart Campus com um campus tradicional demonstrando

\footnotetext{
${ }^{1}$ Neste artigo, os termos cliente e usuário são usados indiscriminadamente para se referir aos indivíduos que acessam a rede sem fio.
} 
a necessidade de inovação que esse ambiente necessita. Uma das formas de inovação seria por meio de sistemas inteligentes capazes de otimizar a qualidade dos serviços nos campi universitários. Com esse intuito, propõe-se uma abordagem para economizar energia da rede sem fio universitária, utilizando um sistema que monitora o funcionamento dos APs da rede, decidindo quais ligar e desligar.

Apostolo et al. [7] utilizam dados coletados, durante seis meses, de um subconjunto de apenas 28 pontos de acesso da rede sem fio da Universidade Federal Fluminense (UFF). O objetivo é prever os períodos de ociosidade da rede através de métodos de aprendizado de máquina. Os autores aplicam métodos de classificação e regressão. Contudo, os autores consideram somente dados de associação a pontos de acesso e ignoram fluxos de dados de usuários ou se estão ativos.

Manweiler et al. propõem um sistema de predição do tempo de estadia de clientes em APs sem fio [12]. A ideia do sistema é aprender assinaturas de um conjunto de clientes inicial no AP e, então, inferir o tempo de permanência de cada cliente em suas proximidades. A predição utiliza diversos sensores nos dispositivos dos clientes, gerando uma matriz de dados que é passada para um classificador Support Vector Machine (SVM) que separa os clientes em classes de comportamento predeterminadas. As predições são geradas em série. De forma semelhante, Lyu et al. [8] propõem um esquema inteligente de controle dinâmico de APs em redes de larga escala com o foco em economia de energia. A estratégia adotada é baseada na previsão da carga dos APs, com base nos dados coletados da rede durante um período de dois meses (carga dos APs, tráfego de dados por AP, entre outros). A proposta realiza uma previsão a cada $24 \mathrm{~h}$ e verifica os momentos em que os APs da rede estarão ociosos, sem nenhuma conexão de usuário. Caso fiquem ociosos por um período de tempo maior ou igual ao estipulado, esses pontos de acesso serão desligados até o momento previsto para uma nova conexão de usuário.

A principal contribuição deste artigo é a otimização energética e a utilização da rede sem perda da capacidade de atendimento aos usuários. Diferentemente de trabalhos anteriores, este artigo foca em uma rede de grande escala e considera fluxos reais dos usuários na rede. A rede da Universidade Federal Fluminense usada como cenário neste artigo conta com 363 pontos de acesso [5], [6]. Na rede considerada, os pontos de acesso alcançam a potência máxima de $5,4 \mathrm{~W}^{2}$. Apenas um ponto de acesso ligado $24 \mathrm{~h}$ por dia em um mês levaria ao consumo máximo de $3,89 \mathrm{kWh} / \mathrm{mês}$. Em toda a rede, os 363 pontos de acesso consumiriam até $1412 \mathrm{kWh} /$ mês. Os métodos de predição de comportamento da rede são importantes para a análise, assim como as medidas adotadas para economia de energia.

\section{Coleta de Dados e Descrição da Rede}

A rede sem fio institucional da Universidade Federal Fluminense (UFF), no campus da Praia Vermelha, é utilizada para

\footnotetext{
${ }^{2}$ A estimação da potência dos pontos de acesso foi realizada em função do modelo TP-Link WR743, modelo mais prevalente na rede da Universidade Federal Fluminense, que opera a uma corrente máxima de 0,6A a 9,0V.
}

a coleta de dados sobre o padrão de uso dos usuários [5], [6]. Essa rede possui 363 pontos de acesso distribuídos pelo campus de maneira não uniforme. Os pontos de acesso permanecem ligados constantemente, sendo um ambiente ideal para a aplicação de métodos de redução de gasto de energia. Os dados coletados visam fornecer padrões de uso em diferentes horários do dia e obter a compreensão da demanda real.

A ferramenta NetFlow é utilizada para a coleta de informações que compõem o conjunto de dados sobre os APs. O conjunto de dados gerado contém todos os fluxos dos clientes e associações entre clientes e APs realizadas durante o período de uma semana. As características do conjunto de dados referente às informações coletadas de cada AP, representado pela característica ap, são packets-forward, bytes-forward, sTime-forward, dur+msec-forward, packets-backward e bytesbackward. A característica "sTime-forward" indica o tempo em que o cliente requisitou um fluxo através do AP indicado. A característica "dur+msec-forward" indica a quantidade de segundos referente à duração do fluxo. As características "packets-forward" e "bytes-forward" são respectivamente a quantidade de pacotes e de bytes enviados, assim como "packets-backward" e "bytes-backward" são a quantidade de pacotes e de bytes recebidos. Há ainda as características "macsta", que indica qual é o endereço MAC do cliente que estabeleceu a associação com o ponto de acesso, e "clientes", que é o número de clientes conectados ao mesmo AP no momento em que ocorre o fluxo.

Desenvolve-se um simulador utilizando a linguagem Python, para reproduzir o comportamento da rede. As simulações são realizadas através da reprodução dos eventos coletados no conjunto de dados da rede. A simulação tem como objetivo identificar os pontos de acesso ociosos e a variação da quantidade de usuários na rede ao longo do dia. A análise dos dados demonstra um padrão de uso da rede com alta demanda no período entre $11 \mathrm{~h}$ e $15 \mathrm{~h}$, tendo as maiores quantidades de usuários do dia, podendo ultrapassar 1.000 usuários simultâneos associados à rede em um dia que não seja fim de semana; e ociosidade no período entre 22 h e 5 h, mantendo uma média de menos de 100 usuários simultâneos associados à rede. Esses períodos coincidem com os horários de atividade do campus em que a rede se encontra. Através dessas informações é possível identificar longos períodos em que os APs não possuem usuários conectados, resultando consumo de energia desnecessário. Com base nesse padrão de utilização, é proposto um novo modelo de gestão de funcionamento dos APs capaz de reduzir o consumo de energia nos períodos com baixa ou nenhuma demanda.

\section{Estratégia de Alta Disponibilidade E DESEMPENHO}

A estratégia proposta visa a redução do consumo de energia por meio de uma gestão eficiente da atividade dos APs, uma vez que, por se tratar de uma rede de grande escala, manter todos os pontos de acesso ativos constantemente resulta em um alto consumo energético. Inicialmente, todos os APs da rede são considerados desligados. Em seguida é selecionado 
um conjunto inicial de APs que serão ligados, denominados APs Base. Após a seleção dos APs Base, o sistema está pronto para analisar as tentativas de associação dos usuários com o propósito de identificar a quais APs cada usuário se associará. A análise se baseia em três informações: (I) AP preferido, ou seja, o ponto de acesso que o usuário consegue estabelecer uma associação com o melhor sinal possível, (II) os APs que estão ligados no momento e (III) a disponibilidade de cada AP para receber novos usuários. Após avaliar as conexões por um período de tempo, duas ações são tomadas em relação aos APs. Na primeira, é verificada a possibilidade de realocação de clientes baseada na qualidade da associação e na disponibilidade dos APs ligados sem prejudicar outros clientes. Novos APs também podem ser ligados para atender à demanda de associação de novos clientes à rede. Na segunda, são identificados, e desligados em seguida, os APs que não possuem clientes associados. Após essa etapa, o processo de análise das associações é reiniciado. Com isso, a estratégia pode ser dividida em duas partes: Seleção de APs Base, e Gerenciamento dos APs, em que os APs são ativados sob demanda de associações de usuários à rede.

\section{A. Seleção de APs Base}

Na primeira etapa, a partir do conhecimento prévio do grafo da rede sem fio, são propostas abordagens diferentes para o conjunto de APs base. As 3 abordagens propostas para a seleção dos APs são: (I) seleção baseada nos APs com o Número Máximo de Vizinhos (NMV); (II) seleção baseada em APs não vizinhos, Priorizando Número de Vizinhos (APnv PNV); e (III) seleção baseada em cliques no grafo da topologia da rede sem fio. As abordagens mencionadas são descritas a seguir:

1) APs com o Número Máximo de Vizinhos: Um vetor auxiliar é criado contendo todos os nós do grafo da rede. Em seguida, o vetor é ordenado em relação à quantidade de vizinhos de cada nó, em ordem crescente. Então, são selecionados todos os nós do grafo que possuem o mesmo número de vizinhos que o nó localizado na última posição do vetor (nó com maior número de vizinhos). Na Figura 1(a), o AP com o maior número de vizinhos possui 4 vizinhos e, assim, todos APs com 4 vizinhos são selecionados como base.

2) APs não vizinhos, Priorizando Número de Vizinhos:

Cria-se um vetor auxiliar contendo todos os nós do grafo ordenado pelo número de vizinhos de cada nó. $\mathrm{O}$ vetor então é percorrido em ordem decrescente, selecionando todos os nós que não sejam vizinhos de nós previamente selecionados. Os nós selecionados formam o conjunto de APs Base. A Figura 1(b) exemplifica a abordagem.

3) Cliques do grafo da rede: O grafo é separado em vetores que representam os cliques, que são subconjuntos desse grafo composto por vértices pertencentes a esse mesmo grafo, contendo os nós que formam o clique correspondente. Os nós dos vetores (cliques) são ordenados, em ordem crescente, em relação ao número de vizinhos. Os cliques são analisados um a um, para que seja eleito para cada clique um AP representante. Os APs eleitos farão parte do conjunto de APs
Base. A verificação dos nós em cada clique ocorre do último ao primeiro elemento do vetor, com a intenção de selecionar o AP representante do clique em questão. Essa verificação consiste em analisar se o nó não possui nenhum vizinho que já foi selecionado como AP representante. Caso atenda a esse critério, o nó em questão se torna o AP representante do clique, caso contrário o próximo nó com mais vizinhos é verificado. A Figura 1(c) mostra um exemplo que segue essa abordagem. O grafo possui 3 cliques logo 3 APs ligados, sendo que eles não são vizinhos entre si.

\section{B. Gerenciamento dos APs}

Visando minimizar o gasto de energia, o gerenciamento de APs consiste em ligar novos APs quando há APs que recebem requisição de associação de novos clientes, mas já estão com sua capacidade no limite, e desligar os APs quando nenhum usuário estiver conectado ao AP, prezando também pela qualidade do sinal entregue ao usuário. Com isso, o gerenciamento dos APs é dividido em dois casos: ligar novos APs e desligar APs.

1) Ligar novos APs: Novos pontos de acesso podem ser ligados em dois cenários distintos: (I) caso o ponto de acesso preferido de um cliente esteja desligado e seus vizinhos indisponíveis, ou (II) caso o AP preferido esteja lotado e um dos seus vizinhos esteja desligado.

No primeiro caso, se o AP preferido estiver desligado, o cliente deverá se conectar a um AP vizinho. Para isso, uma verificação levando em consideração a quantidade de usuários associados, estado e quantidade de vizinhos é feita nos APs vizinhos a fim de encontrar a melhor opção. É escolhido o vizinho com a maior quantidade de associações e que não possua a quantidade máxima de associações. O AP preferido será ligado caso todos os vizinhos possuam o máximo de associações ou estejam desligados. O máximo de associações suportado por um ponto de acesso é considerado igual a 15 associações. Reis et al. mostram que para pontos de acesso com mais de 15 usuários associados e ativos há queda na qualidade de serviço percebida pelos usuários [5].

Caso o AP preferido esteja lotado, seus APs vizinhos são verificados para identificar qual deles possui o maior número de vizinhos. Se todos os vizinhos estiverem indisponíveis, é feita outra verificação para saber se existe algum vizinho desligado, e dentre os vizinhos desligados qual deles possui mais vizinhos. Então, esse vizinho é escolhido para ser ligado.

2) Desligar APs: Normalmente, a única situação em que um ponto de acesso pode ser desligado é caso ele não tenha mais nenhuma associação ativa de usuários. Com isso, adotase uma estratégia para permitir o desligamento de APs que ainda possuam clientes conectados e ainda assim, manter esses usuários conectados à rede. A estratégia consiste em forçar a distribuição dos usuários conectados para outro AP vizinho, permitindo que o primeiro AP fique sem nenhuma associação e possa ser desligado sem prejudicar a qualidade de serviço ou o acesso de usuários à rede ${ }^{3}$.

\footnotetext{
${ }^{3} \mathrm{O}$ procedimento de migração de usuários entre pontos de acesso está fora do escopo deste artigo.
} 
Para realizar a realocação de usuários entre pontos de acesso, os pontos de acesso são analisados tendo como foco identificar a quantidade de vizinhos ligados e se estão com capacidade disponível. Os vizinhos com capacidade disponível e com um sinal de boa qualidade, para clientes no ponto de acesso candidato ser desativado, receberam clientes realocados. Caso após essas operações o AP analisado não possua mais nenhuma associação ativa, ele é desligado.
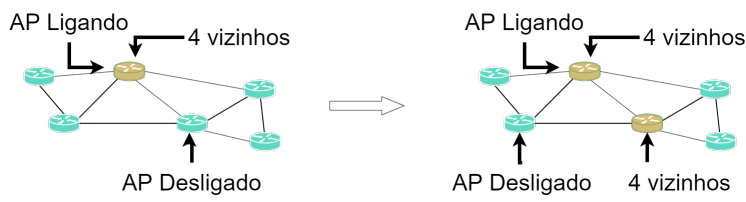

(a) Abordagem de seleção dos APs com o número máximo de vizinhos. Os APs que possuem 4 vizinhos, isto é, o número máximo de vizinhos, são ligados.
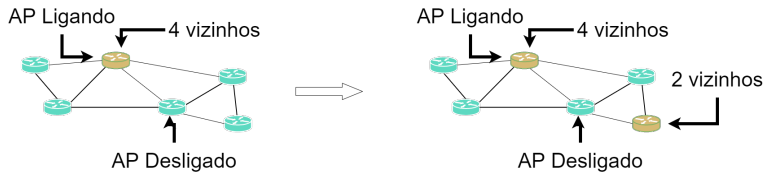

(b) Abordagem de seleção de APs não vizinhos, priorizando aqueles com maior número de vizinhos. Os APs são ligados, um por um, do que possui o maior número de vizinhos até o menor, desde que eles não sejam vizinhos de nenhum que já tenha sido ligado antes.
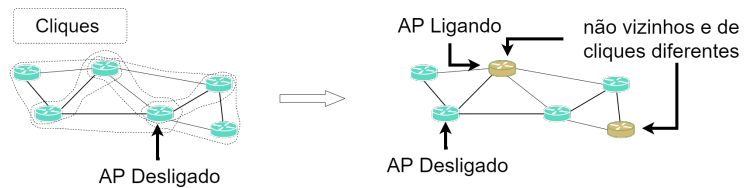

(c) Abordagem de seleção do mais representativo no clique. Os cliques do grafo são identificados e um AP de cada, que não seja vizinho de um AP que esteja ligado, é ligado, desde que nenhum outro AP dentro do mesmo clique já tenha sido ligado.

Figura 1. Exemplos de cada abordagem proposta para selecionar o conjunto de APs base. Representação dos APs ligados em verde.

\section{RESUltados EXPERIMENTAIS}

As três abordagens propostas são comparadas entre si, levando em conta diferentes métricas. Os dados relacionados ao funcionamento de cada abordagem são obtidos através do simulador desenvolvido na linguagem Python. O simulador é capaz de replicar o comportamento da rede com as proposta.

São simuladas a rede em seu funcionamento normal, para a validação do funcionamento do simulador, e a rede funcionando de acordo com as três abordagens propostas. A simulação considera as informações presentes no conjunto de dados obtido, que contém os dados monitorados de 357 $\mathrm{APs}^{4}$ da rede da Universidade Federal Fluminense no período de uma semana. As métricas usadas na comparação das abordagens são: quantidade de APs ligados, quantidade de APs sobrecarregados, quantidade de bytes enviados, quantidade

\footnotetext{
${ }^{4}$ Embora a rede conte com 363 pontos de acesso, seis pontos de acesso estavam indisponíveis no momento da coleta de dados.
}

de bytes recebidos, quantidade de usuários não servidos e quantidade de usuários válidos na rede.

\section{A. Quantidade de APs ligados e Consumo de energia}

Na Figura 2(a), o cenário "Rede pura" representa a rede sem alterações. Nesse cenário, os pontos de acesso ligados são todos os 357 pontos de acesso da rede. Os dados obtidos no cenário "Rede pura" são tomados como base para a comparação e validação do simulador. Dentre as abordagens comparadas, a abordagem NMV apresenta a menor quantidade de APs ligados mantendo aproximadamente 0,6\% de APs ligados durante o período de simulação. Assim, a abordagem NMV apresenta menor gasto de energia em relação à "Rede pura". Os cenários Clique e APnv PNV apresentam, respectivamente, $19 \%$ e $23 \%$ dos APs da rede ligados. O segundo melhor resultado é obtido pela abordagem de cliques, com aproximadamente $18 \%$ a menos de pontos de acesso ligados que a abordagem APnv PNV.

As três abordagens propostas melhoram a eficiência energética da rede avaliada, reduzindo o consumo de energia por meio do desligamento de APs. Considera-se o gasto total de energia por AP aproximadamente igual a 3,89 $\mathrm{kWh} / \mathrm{mês,}$ totalizando $1388,73 \mathrm{kWh} / \mathrm{mês}$ quando todos os APs estão ligados. A estratégia que resulta em menor valor de consumo de energia é a NMV com 11,67 kWh/mês, seguida da abordagem de Cliques com consumo de 264,52 kWh/mês. A abordagem com o maior valor de consumo de energia é o APnv PNV, com 322,87 kWh/mês. Ainda assim, o consumo é reduzido em aproximadamente 4 vezes com a abordagem menos conservadora, APnv PNV.

\section{B. Quantidade de APs sobrecarregados}

A média de pontos de acesso sobrecarregados (APs que possuem mais de 15 usuários associados), por hora, na rede, é muito similar em praticamente todos os cenários simulados. Como mostrado na Figura 2(b), a única abordagem que apresenta uma diferença relevante na média, é a abordagem NMV, com 0,6 APs sobrecarregados por hora. Enquanto o número máximo de APs sobrecarregados nas outras abordagens é 6, na simulação da abordagem NMV, o valor para 2.

\section{Quantidade de bytes recebidos e enviados}

A métrica de bytes enviados e recebidos representa a vazão de tráfego na rede. Uma alta vazão significa que os clientes estão sendo servidos de maneira eficiente e satisfatória. Enquanto que, uma baixa vazão, representa que muitos clientes não estão conseguindo se associar à rede ou que os APs estão sobrecarregados.

Nas Figuras 2(c) e 2(d) pode-se observar a quantidade de bytes trafegados no período simulado, tanto recebidos como enviados. Esses valores apresentam proporções similares quando comparadas. Para os bytes recebidos, o pior resultado se encontra no cenário NMV, com $27 \%$ do valor comparado à simulação referente ao cenário "Rede Pura" na qual não há restrições, uma vez que todos os pontos de acesso estão ligados. A abordagem de Cliques apresenta melhor eficiência 


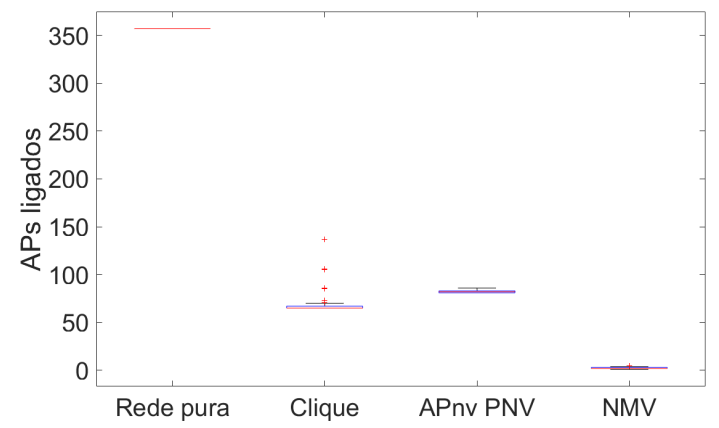

(a) Quantidade de APs ligados na rede ao longo do período de uma semana.

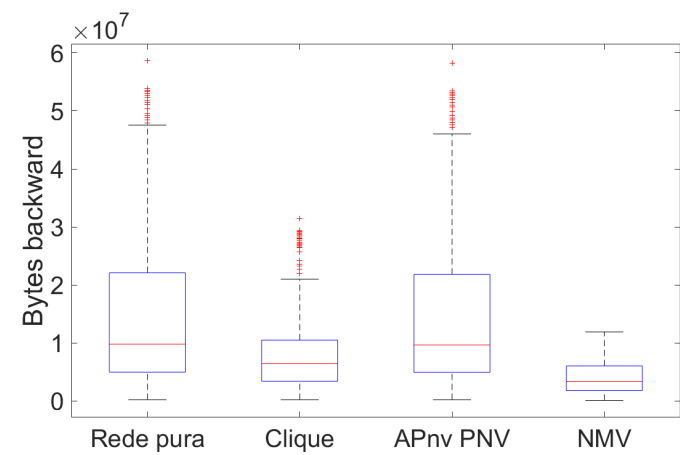

(c) Quantidade total de bytes recebidos ao longo do tempo, em um período de uma semana.

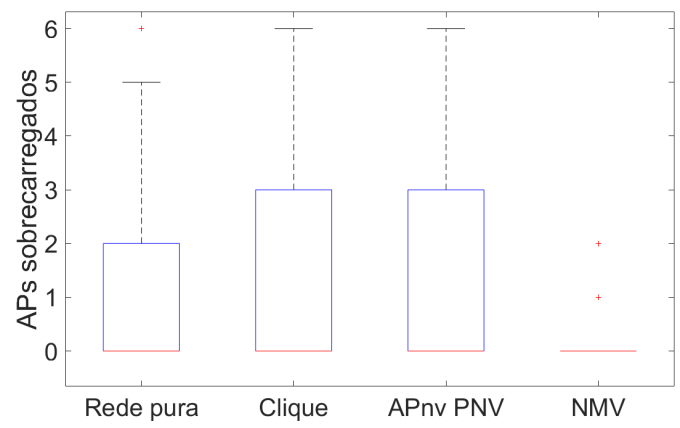

(b) Quantidade de APs sobrecarregados na rede ao longo do período de uma semana.

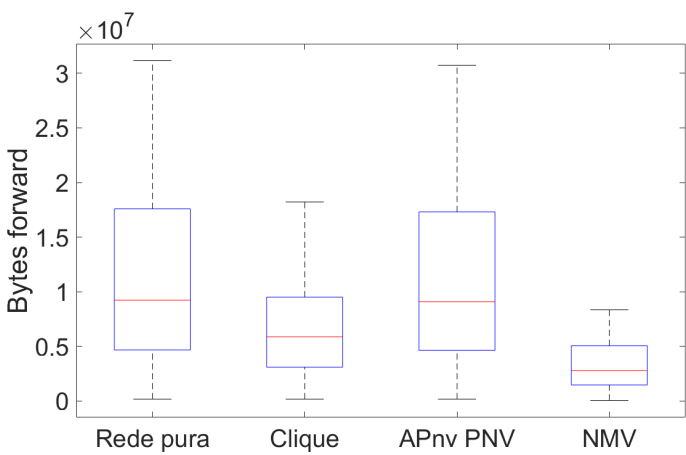

(d) Quantidade total de bytes enviados ao longo do tempo, em um período de uma semana.

Figura 2. Representação da quantidade de APs ligados, quantidade de APs sobrecarregados, quantidade de bytes enviados e quantidade de bytes recebidos para cada abordagem proposta e incluindo a rede em operação normal, sem influência das abordagens propostas.

do que a NMV nesse sentido, alcançando $53 \%$ da quantidade de bytes recebidos. Por fim, a abordagem APnv PNV resulta em $99 \%$ da quantidade de bytes recebidos em relação à quantidade do cenário de "Rede Pura".

Analogamente aos bytes recebidos, para os bytes enviados, o melhor resultado é encontrado para a abordagem APnv PNV, com $98 \%$ da quantidade de bytes enviados na "Rede Pura". As abordagens de Cliques e NMV apresentam respectivamente $58 \%$ e $27 \%$ dos bytes enviados. Assim como na comparação de bytes recebidos, o cenário NMV apresenta os valores mais distantes do cenário "Rede Pura". Assim, apesar da elevada eficiência energética, a qualidade da comunicação experimentada pelo usuário tende a ser ruim.

\section{Quantidade de usuários não servidos e quantidade de usuários válidos na rede}

Juntamente com a quantidade de APs ligados é importante observar a quantidade de usuários não servidos. Essa métrica permite verificar se a abordagem, além de ter um bom desempenho em eficiência energética, consegue atender à demanda dos usuários que tentam se associar a pontos de acesso da rede. Tendo em vista que, quanto menos usuários não servidos, melhor a disponibilidade da rede, observa-se na Figura 3(a) que a abordagem NMV apresenta o pior desempenho dentre as abordagens propostas, com 265 usuários não servidos no período simulado. Comparando as demais abordagens, Clique e APnv PNV, nota-se que mesmo com ambas apresentando um resultado muito melhor que o cenário NMV, ainda há uma considerável diferença entre seus resultados. Enquanto com a abordagem de Cliques 126 usuários não são servidos, com a abordagem APnv PNV apenas 14 usuários não são servidos ao longo do período simulado. A abordagem APnv PNV é 7 vezes melhor do que a de Cliques nessa métrica.

A Figura 3(b) mostra a quantidade de usuários válidos na rede por hora. Usuários válidos são aqueles ativos e associados a pontos de acesso com menos de 15 usuários. Assim, como na análise anterior, os piores resultados ocorrem para a abordagem NMV, que apresenta apenas $25 \%$ dos usuários que tentam conexão com a rede como válidos. Os melhores resultados ocorrem para as abordagens de Cliques e APnv PNV, com a segunda tendo o melhor resultado dentre as abordagens propostas. Para a abordagen APnv PNV, aproximadamente $98 \%$ dos usuários na rede são válidos, o que resulta em uma média de 304, 5 usuários por hora. Na abordagem de Cliques, há $94 \%$ dos usuários válidos, uma média de aproximadamente 290, 76 usuários por hora.

\section{CONCLuSÃo}

Este artigo propôs uma estratégia de gerenciamento de pontos de acesso para uma rede de larga escala, visando manter 


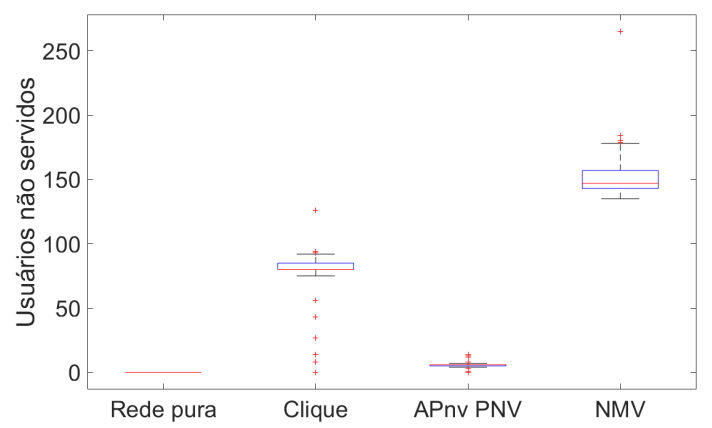

(a) Quantidade total de usuários não servidos ao longo do tempo em um período de uma semana.

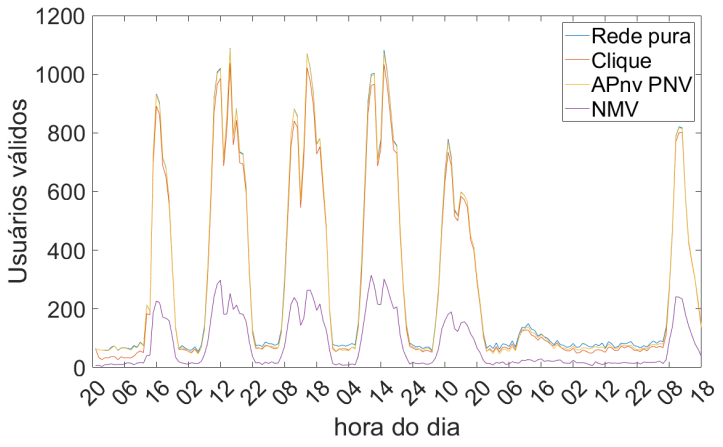

(b) Quantidade de usuários válidos na rede ao longo do período de uma semana.

Figura 3. Quantidade de usuários válidos e de usuários não servidos ao utilizar cada abordagem e durante a operação normal da rede. A abordagem APnv PNV é a que resulta menor número de usuários não servidos dentre as abordagens propostas. O número de usuários válidos é semelhante entre a rede sem interferência e nas abordagens de Clique e APnv PNV.

o nível de disponibilidade da rede e alcançando uma melhor eficiência energética. A estratégia consiste em gerenciar os pontos de acesso da rede, de modo que APs sejam desligados quando não apresentarem nenhum usuário associado. A fim de obter um consumo mais eficiente de energia, foi utilizada uma técnica de realocação de usuários, em que os usuários, que já estão conectados, são remanejados a outros pontos de acesso, com a intenção de reduzir a quantidade de pontos de acesso ligados simultaneamente. Foram propostas e testadas três abordagens para seleção dos conjuntos de pontos de acesso inicialmente ligados: ligação de APs com o Número Máximo de Vizinhos (NMV), ligação dos APs não vizinhos, Priorizando Número de Vizinhos (APnv PNV) e a ligação de APs representantes de cliques no grafo da rede. Os resultados mostram que o desempenho da rede depende fortemente do conjunto de pontos de acesso inicialmente ativados. As abordagens propostas apresentaram melhorias na eficiência energética da rede, quando comparados ao seu funcionamento normal. As abordagens de Cliques e APnv PNV apresentaram um número de usuários válidos na rede que ultrapassou $90 \%$ dos usuários que tentaram se associar à rede. A abordagem de Cliques manteve apenas $19 \%$ APs ligados, enquanto a abordagem APnv PNV apresentou um número de APs ligados $23 \%$ maior que a abordagem anterior. A abordagem APnv PNV apresentou melhor resultado para o número total de usuários servidos, alcançando $98 \%$ dos usuários totais da rede, enquanto mantém 4 vezes menos APs ligados comparada à operação normal da rede, com todos os APs ligados.

Como trabalhos futuros, pretende-se explorar abordagens que resultem em maior economia de energia, reduzindo a quantidade de usuários não servidos. Entre as possíveis alternativas estão as abordagens que utilizam algoritmos de clusterização e redes neurais recorrentes.

\section{AGRADECIMENTOS}

Este trabalho foi realizado com recursos do CNPq, FAPERJ, RNP, CAPES, CGI/FAPESP (2018/23062-5) e Prefeitura de Niterói/FEC/UFF (Edital PDPA 2020). Um agradecimento especial ao Lucas Fauster que contribuiu para a criação do simulador de redes.

\section{REFERÊNCIAS}

[1] Cisco, "Cisco annual internet report (2018-2023) white paper," Cisco, Tech. Rep., 2020

[2] J. Aslan, K. Mayers, J. G. Koomey, and C. France, "Electricity intensity of internet data transmission: Untangling the estimates," Journal of Industrial Ecology, vol. 22, no. 4, pp. 785-798, 2018. [Online]. Available: https://onlinelibrary.wiley.com/doi/abs/10.1111/jiec.12630

[3] S. Lange, J. Pohl, and T. Santarius, "Digitalization and energy consumption. does ict reduce energy demand?" Ecological Economics, vol. 176, p. 106760, 2020. [Online]. Available: http://www.sciencedirect.com/science/article/pii/S0921800919320622

[4] G. Fermino and R. de Moraes, "Uma proposta de protocolo mac com redução de sobrecarga para redes de sensores sem fio," in Anais Estendidos do X Simpósio Brasileiro de Engenharia de Sistemas Computacionais. Porto Alegre, RS, Brasil: SBC, 2020, pp. 25-32.

[5] L. H. A. Reis, L. C. S. Magalhães, D. S. V. de Medeiros, and D. M. Mattos, "An unsupervised approach to infer quality of service for large-scale wireless networking," Journal of Network and Systems Management, vol. 28, no. 4, pp. 1228-1247, 2020.

[6] G. N. N. Barbosa, M. Andreoni Lopez, D. S. V. Medeiros, and D. M. F. Mattos, "An entropy-based hybrid mechanism for Large-Scale wireless network traffic prediction," in 2021 International Symposium on Networks, Computers and Communications (ISNCC), Dubai, United Arab Emirates, May 2021.

[7] G. H. Apostolo, F. Bernardini, L. C. S. Magalhães, and D. C. MuchaluatSaade, "An experimental analysis for detecting wi-fi network associations using multi-label learning," in 2020 International Conference on Systems, Signals and Image Processing (IWSSIP). IEEE, 2020, pp. 423-428.

[8] F. Lyu, L. Fang, G. Xue, H. Xue, and M. Li, "Large-scale full wifi coverage: Deployment and management strategy based on user spatiotemporal association analytics," IEEE Internet of Things Journal, vol. 6, no. 6, pp. 9386-9398, Dec 2019.

[9] L. Bruno, L. Vidigal, D. Guidoni, and F. de Souza, "Uma heurística híbrida para o gerenciamento de recursos na virtualização de funções de rede," in Anais Estendidos do X Simpósio Brasileiro de Engenharia de Sistemas Computacionais. Porto Alegre, RS, Brasil: SBC, 2020, pp. 139-144.

[10] N. Min-Allah and S. Alrashed, "Smart campus—a sketch," Sustainable Cities and Society, vol. 59, p. 102231, 2020. [Online]. Available: http://www.sciencedirect.com/science/article/pii/S2210670720302183

[11] V. Uskov, J. Bakken, A. Pandey, U. Singh, M. Yalamanchili, and A. Penumatsa, "Smart university taxonomy: Features, components, systems." Springer Link, 2016.

[12] Manweiler, Santhapuri, Choudhury, and Nelakuditi, "Predicting length of stay at wifi hotspots." Proceedings IEEE INFOCOM 2013, 2013. 